\title{
A New Scenario for Negative Functional Magnetic Resonance Imaging Signals: Endogenous Neurotransmission
}

\author{
Yen-Yu I. Shih, ${ }^{1 *}$ Chiao-Chi V. Chen, ${ }^{1 *}$ Bai-Chuang Shyu, ${ }^{1}$ Zi-Jun Lin, ${ }^{1}$ Yun-Chen Chiang, ${ }^{1,2}$ Fu-Shan Jaw, ${ }^{2}$ \\ You-Yin Chen, ${ }^{3}$ and Chen Chang ${ }^{1}$ \\ ${ }^{1}$ Institute of Biomedical Sciences, Academia Sinica, Nankang, Taipei, Taiwan 115, Republic of China, ${ }^{2}$ Institute of Biomedical Engineering, National Taiwan \\ University, Taipei, Taiwan 106, Republic of China, and ${ }^{3}$ Department of Electrical and Control Engineering, National Chiao-Tung University, Hsinchu, \\ Taiwan 300, Republic of China
}

Functional magnetic resonance imaging (fMRI) has revolutionized investigations of brain functions. Increases in fMRI signals are usually correlated with neuronal activation, but diverse explanations have been proposed for negative fMRI responses, including decreases in neuronal activity, the vascular-steal effect, and large increases in oxygen consumption. These possible scenarios, although encompassing a wide range of potential neurovascular responses, cannot yet be used to interpret certain types of negative fMRI signals. Recent studies have found that intravenous injection of dopamine $\mathrm{D}_{2}$ receptor (D2DR) agonist reduced the hemodynamic responses in the caudate-putamen $(\mathrm{CPu})$; however, whether endogenous dopaminergic neurotransmission contributes to fMRI signals remains obscure. Since it has been suggested that the D2DR is involved in pain modulation, and the CPu shows equivocal fMRI signals during noxious stimulation, the present study established an animal model based on graded electrical stimulation to elicit different levels of nociception, and aimed to determine whether nociception-induced endogenous dopaminergic neurotransmission is sufficient to generate negative fMRI responses. Our results from cerebral blood volume (CBV)-weighted fMRI, Fos immunohistochemistry, and electrophysiological recording demonstrated a salient bilateral $\mathrm{CBV}$ decreases associated with heightened neuronal activity in the $\mathrm{CPu}$ induced by unilateral noxious electrical stimulation. In addition, preinjection of D2DR antagonist reduced the observed CBV decreases. Our findings reveal the role of the D2DR in regulating striatal vascular responses and suggest that endogenous neurotransmission-induced CBV decreases underlie negative fMRI signals. Hence, the influence of endogenous neurotransmission should be considered when interpreting fMRI data, especially in an area involved in strong vasoactive neurotransmission.

\section{Introduction}

Blood oxygenation level-dependent (BOLD) functional magnetic resonance imaging ( $\mathrm{fMRI}$ ) has become a pivotal method for noninvasive measuring of brain functions (Ogawa et al., 1990), and has ushered in a new era of neuroscience research. Positive fMRI signals are usually correlated with neuronal activation; however, the negative responses, resulting in various confounding effects on mapping brain function and dysfunction, are lesswell understood. Negative BOLD signals have been attributed to three main possible scenarios: decreases in CBF due to the suppression of neuronal activity (Shmuel et al., 2002, 2006; Devor et al., 2007), the vascular-steal effect (Harel et al., 2002; Shmuel et al., 2002), and increases in oxygen metabolism that exceed the arterial blood compensation (Schridde et al., 2008). Nevertheless,

Received July 23, 2008; revised Dec. 30, 2008; accepted Jan. 2, 2009

We acknowledge technical support from the Functional and Micro-Magnetic Resonance Imaging Center supported by the National Research Program for Genomic Medicine, National Science Council, Taiwan, Republic of China, NSC97-3112-B-001-009. We also thank Dr. Allan Basbaum for his kind input.

*Y.-Y.I.S. and C.-C.V.C. contributed equally to this work.

Correspondence should be addressed to either of the following: You-Yin Chen, Department of Electrical and Control Engineering, National Chiao-Tung University, 1001 University Road, Hsinchu, Taiwan 300, Republic of China, E-mail: irradiance@so-net.net.tw; or Chen Chang, Institute of Biomedical Sciences, Academia Sinica, No. 128, Section 2, Academia Road, Nankang, Taipei, Taiwan 115, Republic of China, E-mail: bmcchen@ibms.sinica.edu.tw.

D01:10.1523/JNEUROSCI.3447-08.2009

Copyright $\odot 2009$ Society for Neuroscience $\quad$ 0270-6474/09/293036-09\$15.00/0 the origin of certain types of negative fMRI responses is still unclear. Previous studies have shown that intravenous injection of drugs or specific receptor agonists can mediate fMRI signals (Chen et al., 2005; Choi et al., 2006), but whether endogenous neurotransmission is sufficient to alter fMRI data remains obscure. If endogenous neurotransmission accompanies significant changes in hemodynamic responses, caution is necessary when interpreting fMRI data obtained when performing a specific task that evokes vasoactive neurotransmission. Therefore, revealing the role of endogenous neurotransmission in fMRI responses is of considerable importance.

Unexplainable negative hemodynamic responses have been observed in the caudate-putamen $(\mathrm{CPu})$ following noxious stimulation, which is of long-standing interest to neuroscientists and neurologists (Morrow et al., 1998; Zhao et al., 2008a). We hypothesized that endogenous dopaminergic neurotransmission can lead to these confounding effects since the dopamine $D_{2}$ receptor (D2DR) has been proposed as being involved in regulating both pain behaviors (Magnusson and Fisher, 2000; Mansikka et al., 2005) and vascular responses (Choi et al., 2006). It has also been suggested that D2DR availability is positively correlated with the response to pain in both healthy subjects (Pertovaara et al., 2004; Scott et al., 2006) and patients with chronic pain syndrome (Hagelberg et al., 2004). Consequently, both understanding the complex regulation of the vascular activity by endogenous 
dopaminergic neurotransmission and clinical investigations of pain modulation require a better understanding of underlying fMRI signals.

The aims of the present study were to determine whether endogenous dopaminergic neurotransmission can influence fMRI data and to elucidate the role of the $\mathrm{CPu}$ in nociceptioninduced cerebral hemodynamics. To address these questions, graded electrical stimulation was applied to the rat forepaw to elicit responses to different degrees of noxious input, and cerebral blood volume (CBV)-weighted fMRI was used to detect the corresponding vascular responses, since this method can eliminate the contribution of oxygen metabolism to negative BOLD responses (Mandeville et al., 1998; Keilholz et al., 2006; Van et al., 2006). Combined with data from Fos immunohistochemistry and electrophysiological recordings, the findings suggest that endogenous dopaminergic neurotransmission is an important contributor to negative or reduced hemodynamic responses. Therefore, the influence of endogenous neurotransmission should be considered as a novel putative mechanism underlying negative fMRI signals.

\section{Materials and Methods}

Subjects. A total of 52 adult male Wistar rats (8-10 weeks old; weighing 250-300 g; National Laboratory Animal Center, Taiwan) were used in the present study. The animals were housed in a well-controlled environment with a $12 \mathrm{~h}$ light/dark cycle and controlled humidity and temperature. Rats were triple-housed in plastic cages with ad libitum access to food and water. All experimental procedures were approved by the Institute of Animal Care and Utilization Committee at Academia Sinica, Taipei, Taiwan.

Histological experiments: Fos immunohistochemistry of the spinal cord. A group of 25 animals were used for spinal Fos immunohistochemistry. The effects of electrical stimulation at three intensities (5, 20, and 60 V) were compared with no stimulation (control) serving as a negative control and with formalin injection (formalin) serving as a positive control, yielding five treatment groups. Two hours after treatment, the rats were perfused transcardially with saline followed by $4 \%$ paraformaldehyde. The cervical and lumbar spinal cords were gentled removed, postfixed in $4 \%$ paraformaldehyde for $24 \mathrm{~h}$, immersed in $20 \%$ sucrose, and then cryosectioned at $50 \mu \mathrm{m}$. To begin the immunostaining, the spinal sections were pretreated with PBS containing $0.3 \% \mathrm{H}_{2} \mathrm{O}_{2}$ and $0.1 \%$ sodium azide. Sections were then incubated in anti-Fos (Ab5, Oncogene; 1:50,000) for $48 \mathrm{~h}$ at $4^{\circ} \mathrm{C}$ followed by incubation in biotinylated goat antirabbit IgG (Jackson Immunoresearch; 1:1000) for $4 \mathrm{~h}$ at room temperature. The sections were exposed to avidin-biotin-peroxidase complex (ABC Elite kit, Vector; 1:500) for $3 \mathrm{~h}$ at room temperature, and then stained in $0.025 \% \mathrm{DAB}$ (diaminobenzidine), $1.5 \%$ nickel ammonium sulfate, $0.024 \% \mathrm{H}_{2} \mathrm{O}_{2}$ in $0.05 \mathrm{M}$ Tris buffer for 5-10 min until the desired dark-purple color had developed. The sections were then washed, mounted on coated slides, dehydrated, and coverslipped with DPX.

The Fos-immunoreactive (Fos-ir) cells were analyzed under a light microscope (BX51, Olympus). Six sections from each of the cervical and lumbar spines distributed rostrocaudally were used for quantification. The dorsal horns (laminas I to V) of the cervical and lumbar spines were selected as the regions of interest (ROIs). Cells with darkpurple or black deposits in the nucleus were considered to be Fos-ir. Fos expression was quantified by manually counting the number of Fos-ir cells expressed in the region. For statistical analysis, two-way ANOVAs $(n=15)$ were used to assess differences in Fos expression between the five treatment groups. The between-subjects factor was the treatment, and the within-subject factor was the side of the spine. The significance level was set at $p<0.05$.

fMRI experiments: animal preparation. On the experiment day, each rat was initially anesthetized with 3\% isoflurane. A PE-50 catheter was inserted into the right femoral vein for administering anesthetic and the contrast agent. Alpha-chloralose $(70 \mathrm{mg} / \mathrm{kg})$ prepared by dissolving in
$0.9 \%$ saline and $10 \%$ polyethylene glycol was then administered for subsequent anesthesia. The femoral artery was also catheterized to monitor the arterial blood gas parameters (OPTI CCA Blood Gas Analyzer, Osmetech) and the arterial blood pressure (IX-214, iWorx). The rats were allowed to breathe spontaneously on their own so as to avoid the possible influence of tracheotomy on brain nociceptive processing; this also kept the physiological autoregulation intact while studying nociception without external intervention (Liu et al., 2004; Lowe et al., 2007; Shih et al., 2008a, 2008b, 2008c). The rat was fixed in a customized head holder by two ear bars and an incisor fixer. The body temperature was maintained at $37^{\circ} \mathrm{C}$ using a warm-water blanket, and the end-tidal $\mathrm{CO}_{2}$ concentration was continuously monitored using a respiration-ventilation monitor (Capnomac Ultima, Datex-Ohmeda). The averaged end-tidal $\mathrm{CO}_{2}$ concentration was $3.0-3.5 \%$. The data were acquired when the baseline $p \mathrm{CO}_{2}$ was lower than $55 \mathrm{mmHg}$ and $p \mathrm{O}_{2}$ was $>110 \mathrm{mmHg}$. The mean arterial blood pressure before stimulation was $115-125 \mathrm{mmHg}$, and this increased during electrical stimulation by $5-25 \mathrm{mmHg}$; such changes are lower than the threshold for inducing nonspecific activation (Tuor et al., 2002). For the CBV-weighted fMRI study, superparamagnetic iron oxide nanoparticles (SPIOs; Resovist, Schering) were administered intravenously to the rat at a dose of $30 \mathrm{mg} \mathrm{Fe} / \mathrm{kg}$ as a contrast agent. These SPIOs are slowly eliminated from the blood pool (blood half-life of 2.4-3.6 h). During neuronal activation, increases in regional CBV would increase the quantity of SPIOs, leading to a lower MR signal intensity.

Electrical stimulation was then applied to the rat forepaw. Two needle electrodes were inserted under the skin of the right forepaw: one between the first and second digits and the other between the third and fourth digits. These electrodes were then fixed with surgical tape and the stimulation was confirmed by observing digit twitching. Image acquisition commenced once the rat had been conditioned as described above. Graded electrical stimulation at 5, 20, and $60 \mathrm{~V}$ with a $3 \mathrm{~Hz}$ square wave and a $0.5 \mathrm{~ms}$ pulse duration was applied to five rats by an constantvoltage stimulator (Digitimer), with trials separated by at least $10 \mathrm{~min}$ to avoid adaptation. The noxious $60 \mathrm{~V}$ electrical stimulation of the rat forepaw with a pair of needle electrodes was quantified using a compound ammeter, which showed that the delivered current was $\sim 15 \mathrm{~mA}$. This current level is known to activate $\mathrm{A} \delta$ and $\mathrm{C}$-fibers and induce nociception (Tuor et al., 2000; Chang and Shyu, 2001; Liu et al., 2004; Zhao et al., 2008b).

fMRI experiments: pharmacological MRI. A D2DR antagonist, eticlopride (Sigma-Aldrich), was used to study nociception-induced CBV decreases (as described in the Results) since previous studies have demonstrated that injection of D2DR agonist causes CBV decreases (Choi et al., 2006) whereas injection of D2DR antagonist increases the amphetamineinduced CBV increases (Chen et al., 2005). Five rats received $60 \mathrm{~V}$ right forepaw stimulation followed by CBV-weighted fMRI. Eticlopride was then administered intravenously at $1 \mathrm{mg} / \mathrm{kg}$. No significant alteration on physiological condition was observed following eticlopride administration. After allowing $10 \mathrm{~min}$ for the eticlopride to take effects via the circulation, identical imaging protocols were performed with the same stimulation parameters described above.

fMRI experiments: data acquisition. For fMRI experiments, images were captured using a 4.7-T Bruker Biospec 47/40 spectrometer with a 72 $\mathrm{mm}$ volume coil used as the RF transmitter and a quadrature surface coil placed on the head as the receiver. $\mathrm{A}_{2}$-weighted pilot image was taken in the axial and mid-sagittal plane to localize the anatomical position by identifying the anterior commissure (bregma $-0.8 \mathrm{~mm}$ ). Since the rat head was fixed firmly to the stereotaxic frame with two ear bars in an identified interaural line through the external auditory meatus, the image appeared to be horizontal. If rotation occurred, the stereotaxic frame in the magnetic bore was adjusted to correct the angle until the scanned image showed no rotation. After localization, 3 slices with a FLASH sequence comprising 60 time frames was used for CBV fMRI experiments with a repetition time of $150 \mathrm{~ms}$, echo time of $20 \mathrm{~ms}$, flip angle of $22.5^{\circ}$, field of view of $2.56 \times 2.56 \mathrm{~cm}^{2}$, slice thickness of $1.5 \mathrm{~mm}$, number of excitation of 1 , acquisition matrix of $128 \times 64$ (zero-filled to $128 \times$ 128 ), and temporal resolution of $9.6 \mathrm{~s}$. The centers of the three slices were located at bregma $+0.7,-0.8$, and $-2.3 \mathrm{~mm}$. An OFF-ON-OFF paradigm was used to detect the responses to electrical stimulation, with the 
A

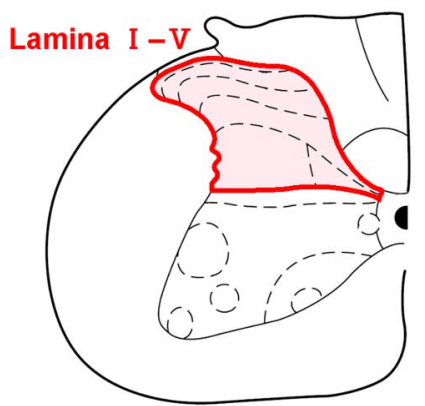

B

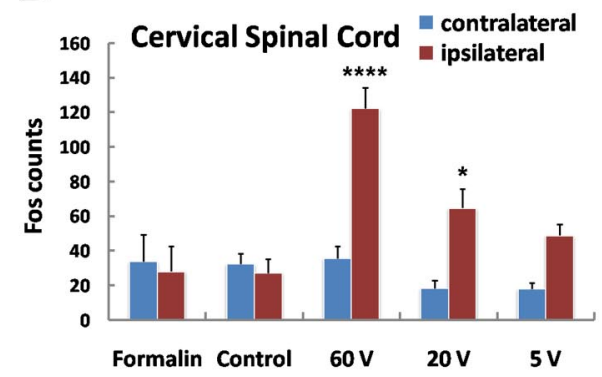

C

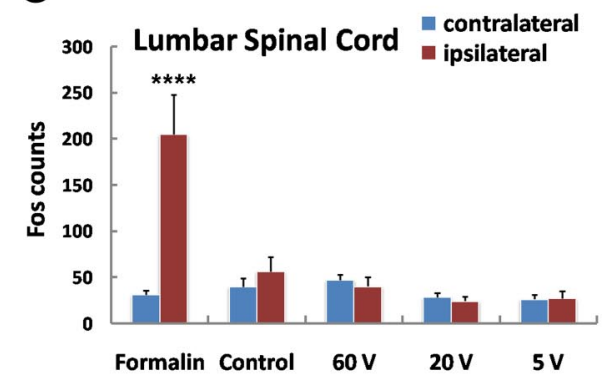

D

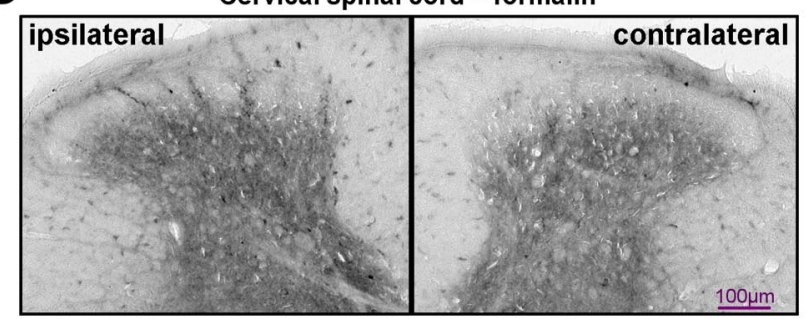

F

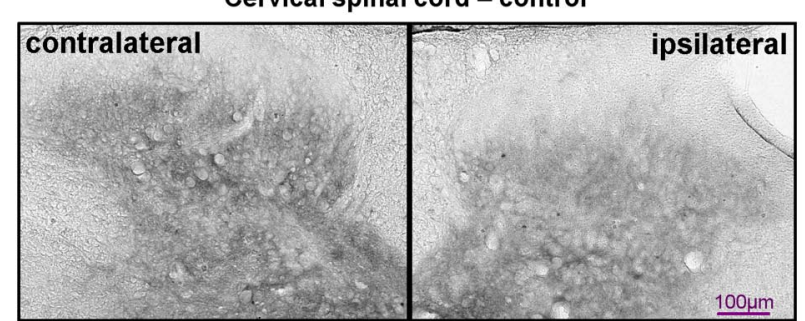

E

Cervical spinal cord $-60 \mathrm{~V}$

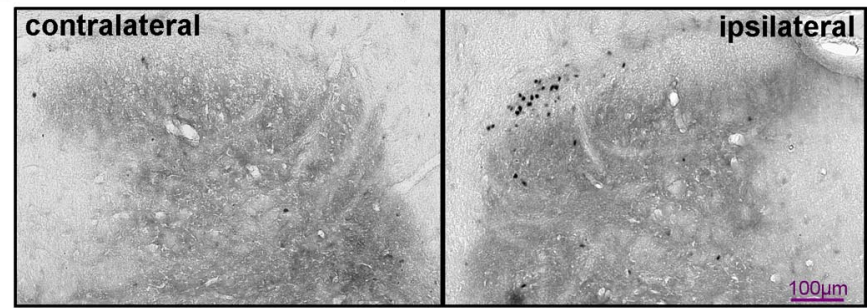

G

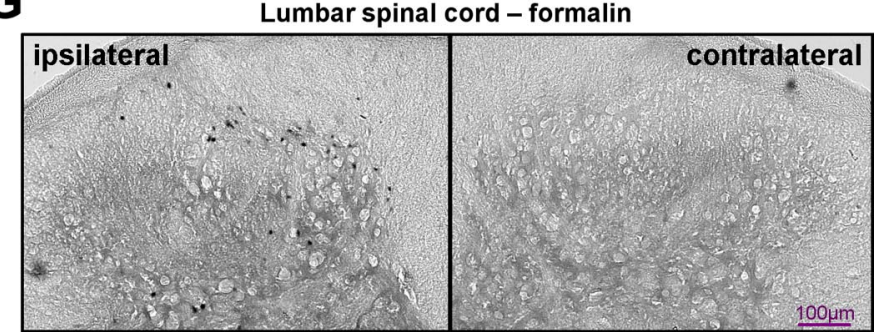

Figure 1. Stimulation noxiousness was quantified by spinal Fos immunohistochemistry in the dorsal horn region. $\boldsymbol{A}$, The ROl used for Fos quantification in the cervical spinal cord. $\boldsymbol{B}$, Electrical stimulation to the right forepaw induced Fos expression in the ipsilateral dorsal horn of the cervical spine. C, Formalin injection into the left hindpaw induced Fos expression in the ipsilateral dorsal horn of the lumbar spine. $D-G$, Photomicrographs of Fos-stained sections from the cervical spine of the formalin, $60 \mathrm{~V}$, and control groups, and the lumbar spine of the formalin group, respectively. Fos protein appeared to be specifically located in spinal lamina I-V, suggesting the nociceptive nature of the electrical stimulation at $60 \mathrm{~V}$ and formalin injection. ${ }^{*} p<0.05{ }^{* * * *} p<0.0001$, significant. Error bars represent SEM values.

first and last 20 frames categorized as baseline, and the middle 20 frames collected during stimulation.

$f M R I$ experiments: $f M R I$ data analysis. Images were analyzed using a custom-built ISPMER data processing system (Shih et al., 2007, 2008ac). The signal-to-time curves were created by standardizing the mean signal intensity of a region at each time point to percentage changes relative to that at the prestimulus time point. The ROI selection was consistent with a rat brain atlas (Paxinos and Watson, 1998) for a section at bregma $+0.7 \mathrm{~mm}$. Correlation coefficient (CC) analysis was then performed on a pixel-by-pixel basis to correlate MR signal changes and electrical stimulation. Each time course was calculated with an OFFON-OFF paradigm and a CC threshold of $r= \pm 0.4$ was used to generate the correlation maps. These maps were then spatially transformed into a rat-atlas reference domain to obtain the incidence images by spatial averaging. The incidence images presented here represent the averaged correlation maps of an experimental group, in which higher and lower pixel values indicate larger and smaller numbers of rat responses consistent with the stimulation, corresponding to CBV decreases and increases, respectively.

For statistical analysis, the number of pixels with signal changes that differed significantly from the baseline values for each of the graded electrical stimulations were quantified and compared statistically. The signals were compared between before (time frames 1-20) and during (time frames 21-40) electrical stimulation using two-tailed paired $t$ tests, with $p<0.05$ considered indicative of a significant difference. A repeated-measures ANOVA $(n=5)$ was used to examine whether the number of pixels showing MR signal changes differed with the stimulation intensity, with the significance level set at $p<0.05$.
Histological experiments: Fos immunohistochemistry of the CPu. Fos immunostaining was applied to brain tissues from the $60 \mathrm{~V}$, control, and formalin groups as described above. Fos expression in the $\mathrm{CPu}$ was determined by the summed number of Fos-ir cells from three axial sections (bregma $+0.7,-0.8$, and $-2.3 \mathrm{~mm}$ ), which covered the CPu from rostral to caudal. The statistical analysis method was similar to that used to quantify spinal Fos.

Electrophysiological recording. Seven rats prepared in the same way were used for the electrophysiological experiment. The rats were implanted stereotaxically with pairs of eight microwire electrode arrays (no. 15140/13848, $50 \mu \mathrm{m}$ in diameter; California Fine Wire) in the bilateral $\mathrm{CPu}$ (see Fig. $5 C$ ). The coordinates used were $0.7 \mathrm{~mm}$ anterior to the bregma, $\pm 2.5 \mathrm{~mm}$ lateral to the midline, and $4.5-5.2 \mathrm{~mm}$ below the cortical surface (Paxinos and Watson, 1998). The recording protocol involved $50 \mathrm{~s}$ of rest alternated with $50 \mathrm{~s}$ of electrical stimulation $(60 \mathrm{~V}, 3$ $\mathrm{Hz}$ square wave of $0.5 \mathrm{~ms}$ duration). Neuronal activity was recorded using a 16-channel multichannel data acquisition processor (MAP, Plexon). Extracellular signals from the electrodes were amplified, bandpass filtered (spike preamp filter: $0.45-5.00 \mathrm{kHz}$, gain: $15,000-20,000$ ), and sampled at $25 \mathrm{kHz}$. The action potentials were resolved using a statistical threshold criterion (Donoho and Johnstone, 1994) for spike detection, where the threshold value $\left(T_{h}\right)$ was selected as $T_{h}=4 \mathrm{o}_{n}$, where $o_{n}$ is an estimate of the $\mathrm{SD}$ of the background signal according to

$$
\sigma_{n}=\operatorname{median}\left\{\frac{|x|}{0.6745}\right\},
$$

where $x$ is the signal comprising spikes and noise. 


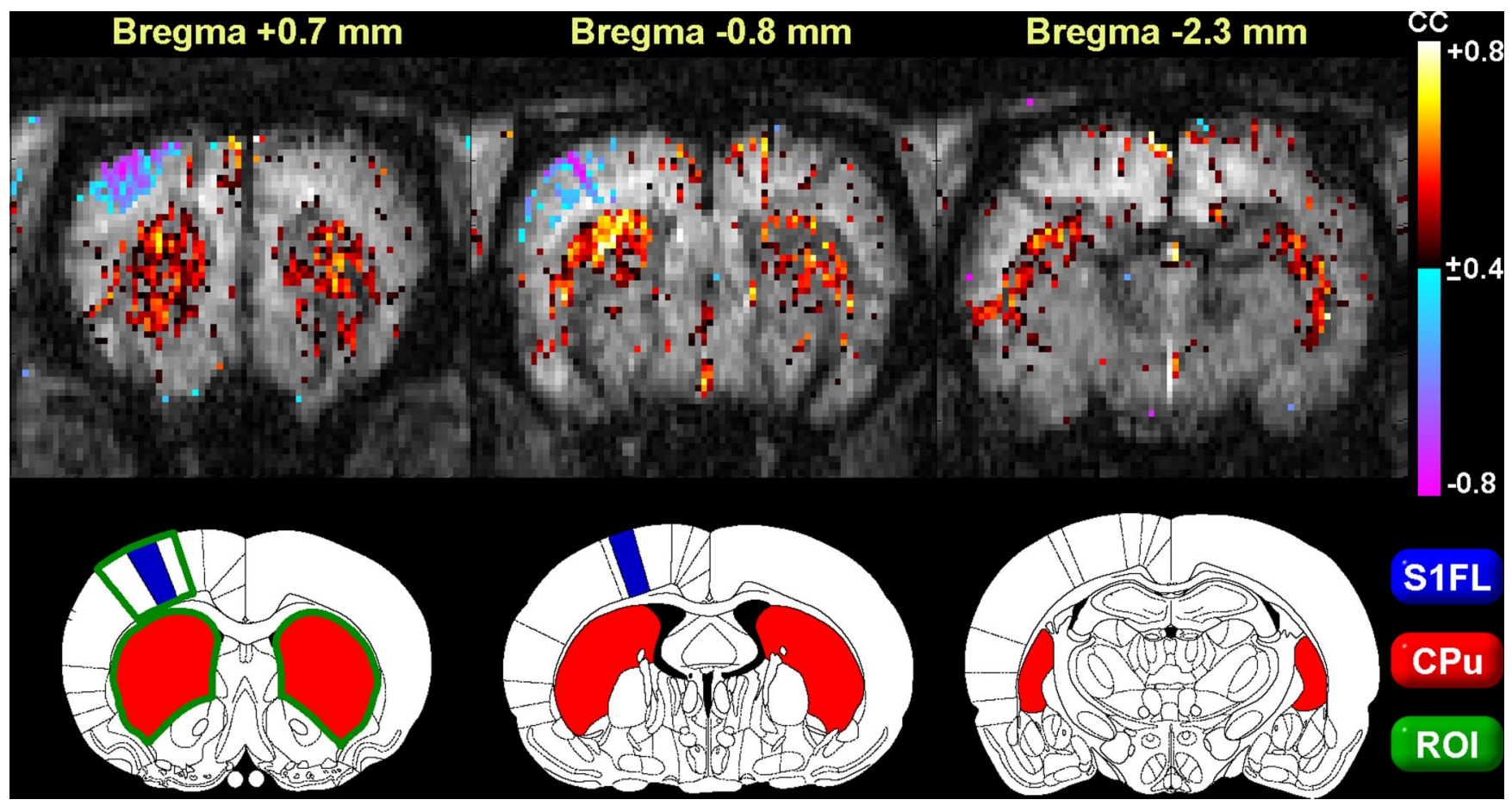

Figure 2. Nociception-induced CBV decreases in the CPu. A 60 V electrical stimulation was applied to the right forepaw of a rat followed by CBV-weighted fMRI with data acquired at bregma $+0.7,-0.8$, and $-2.3 \mathrm{~mm}$. Data were analyzed using a CC method with a threshold of $r= \pm 0.4$. Positive (CBV decreases) and negative (CBV increases) correlations are indicated by hot and cold colors, respectively. The anatomical reference based on the rat brain atlas and the corresponding ROI positions for subsequent analysis are shown in the lower row. Clear CBV increases were evident in the contralateral S1FL, whereas strong CBV decreases were evident in the bilateral CPu.

The spike trains were then analyzed using NeuroEXplorer (Nex Technologies) to determine the neuronal firing rate, and the two-tailed paired $t$ test was used to examine the change in firing rate in the $\mathrm{CPu}$ between before and during stimulation. After recording, the animals were perfused with $4 \%$ paraformaldehyde, and then debrained. The brain tissues sectioned at $50 \mu \mathrm{m}$ were stained with $0.1 \%$ cresyl violet to verify the location of the implanted electrodes.

\section{Results}

Validation of the nociceptive nature of electrical stimulation The nociceptive nature of electrical stimulation was validated using Fos immunohistochemistry of the spinal cord. Electrical stimulation was applied to the right forepaw, and formalin (positive control) was injected into the left hindpaw. The control animals were sham-operated without electrical stimulation applied. The sample size in each group was five, and the ROI used for Fos quantification is shown in Figure $1 A$. Electrical stimulation of $60 \mathrm{~V}$ induced significantly more Fos-ir cells in the ipsilateral dorsal horn of the cervical spine, whereas the formalin injection induced more in the ipsilateral dorsal horn of the lumbar spine (Fig. $1 B, C$ ). Two-way ANOVAs $(n=25)$ indicated that these treatments resulted in differences in the expression of Fos-ir cells, with the effects varying with the side of the spinal cord (all $p<0.001)$. In the cervical spine, Fisher's post hoc tests revealed that the induced Fos expression was greatest for $60 \mathrm{~V}$ stimulation, with the expression being significantly higher on the ipsilateral side $(p<0.001)$. Although the increase in Fos-ir cells induced by stimulation at 20 and $5 \mathrm{~V}$ was not significant when compared with that induced by formalin and in controls $(p>0.05)$, the ipsilateral expression was greater than the contralateral expression $(p<0.05)$. The Fos expression in the lumbar spine was significantly higher in the formalin group than in the control, 60 $\mathrm{V}, 20 \mathrm{~V}$, and $5 \mathrm{~V}$ groups (all $p<0.01$ ), with the increase being higher on the ipsilateral side. The specific increases in Fos expres- sion in the spinal dorsal horn suggested that the electrical stimulation and formalin injection were nociceptive in nature. Photomicrographs of the Fos-ir cervical spine for the formalin, $60 \mathrm{~V}$, and control groups are shown in Figure $1 D, \mathrm{E}$, and $\mathrm{F}$, respectively, and the lumbar spine for the formalin group is shown in Figure $1 G$.

\section{CBV changes during graded electrical stimulation}

$\mathrm{CBV}$ changes in the $\mathrm{CPu}$ in response to electrical stimulation were assessed by CBV-weighted fMRI. Figure 2 shows the results following $\mathrm{CC}$ analysis for $60 \mathrm{~V}$ stimulation to the right forepaw. Three axial $\mathrm{T}_{2}{ }^{*}$-weighted images at bregma $+0.7,-0.8$, and -2.3 $\mathrm{mm}$ were dynamically acquired to identify changes in CBV. The color-coded regions in Figure 2 correspond to areas with $\mathrm{CBV}$ changes whose activation patterns were positively or negatively correlated with the OFF-ON-OFF stimulus paradigm, where positive and negative correlations are associated with CBV decreases and increases, respectively. The figure indicates that electrical stimulation induced intense CBV decreases mainly in the $\mathrm{CPu}$ across the three slices, which was accompanied by CBV increases in the contralateral forelimb area of primary somatosensory cortex (S1FL).

Figure 3 presents the $\mathrm{CC}$ of the five cases averaged on a pixel-by-pixel basis and color-coded, which shows that the $\mathrm{CBV}$ increases was induced in the S1FL region for all the stimulation intensities. However, the $\mathrm{CPu}$ exhibited differing degrees of CBV decreases that became more widespread with increasing stimulation intensity. Figure $4 \mathrm{~A}$ illustrates the signal-to-time curves of the S1FL and the CPu over the 60 time points (corresponding to an imaging period of $\sim 9.6 \mathrm{~min}$ ) at each of the graded intensities. The curves indicated that the $\mathrm{MR}$ signals in the $\mathrm{CPu}$ increased immediately during electrical stimulation, reaching a plateau within $1 \mathrm{~min}$, and staying ele- 


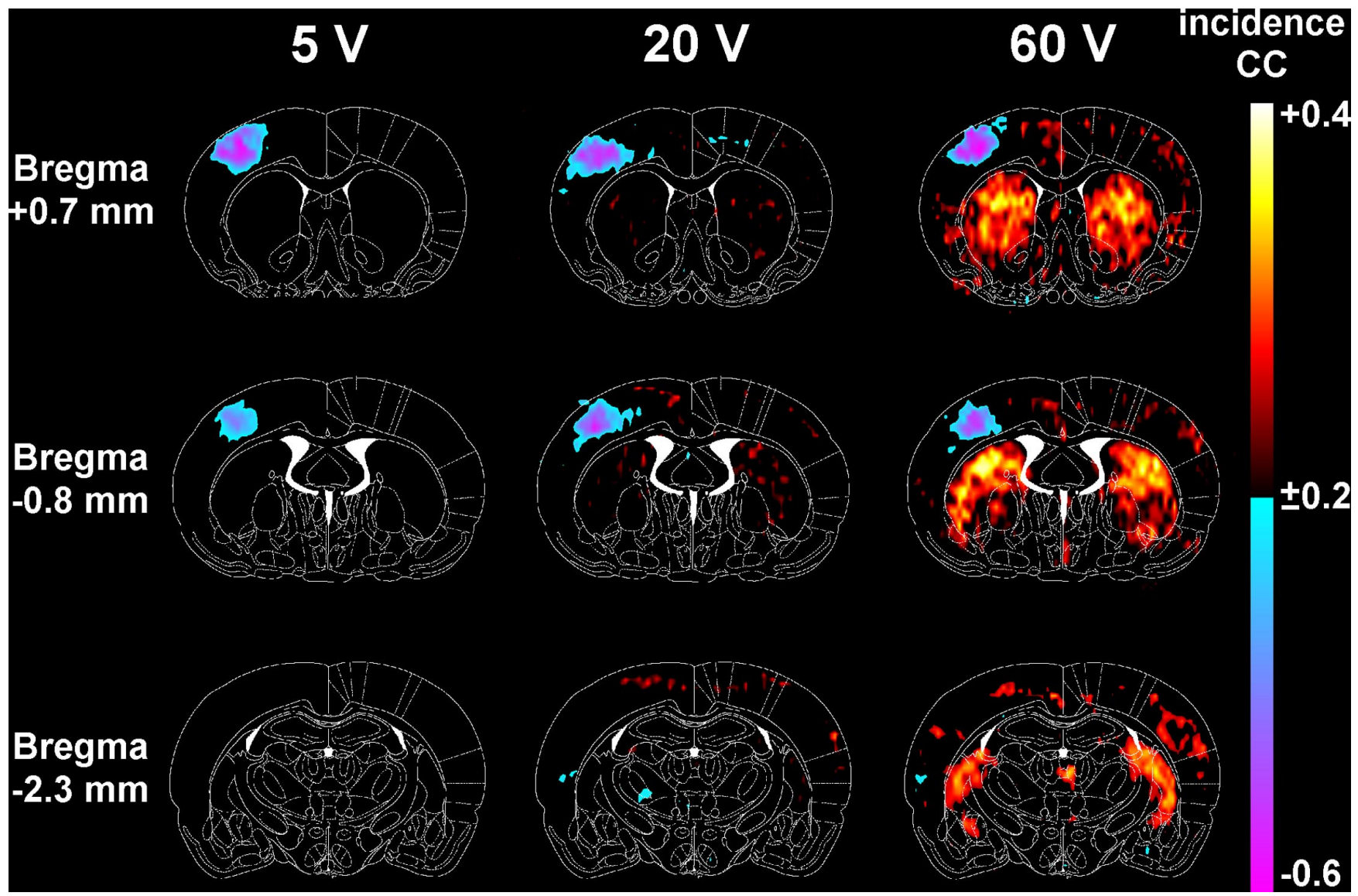

Figure 3. CBV changes in five rats subjected to 5, 20, and $60 \mathrm{~V}$ forepaw electrical stimulation followed by CBV-weighted fMRI. CC data were used to generate the incidence images. The rat brain atlas was used as a template for spatial normalization, and the correlation maps of each rat were then averaged on a pixel-by-pixel basis to display the incidence responses, where higher and lower pixel values indicate decreases and increases in averaged CBV responses, respectively. These images show that the CPu did not respond to $5 \mathrm{~V}$ forepaw electrical stimulation, but that the degree of CBV decreases increased for higher stimulation intensities.

vated for the remaining stimulation period. The changes in MR signal intensity at the plateau (defined as the averaged signal values over a 2 -min period beginning 1 min after stimulus initiation) in the contralateral S1FL for stimulation at 5, 20 , and $60 \mathrm{~V}$ were $-24.86 \pm 1.74 \%,-27.69 \pm 3.34 \%$, and $-24.17 \pm 3.71 \%$ (mean $\pm \mathrm{SD}$ ), respectively, whereas those in the $\mathrm{CPu}$ were $1.38 \pm 0.88 \%, 6.59 \pm 0.89 \%$, and $10.15 \pm$ $1.13 \%$. The CBV response of an area was quantified by the number of responsive pixels in the region. Responsive pixels were defined as those with significant signal changes from baseline as detected by two-tailed paired $t$ tests with a significance level of $p<0.05$. Note that correlation and incidence maps provide the spatial distribution of the induced response in the rat brain, while the quantification of responsive pixels indicates both the level of significant signal changes and the spatial extent of the response induced by the stimulation. Referring to Figure $4 B$, repeated-measures ANOVA $(n=5)$ indicated that electrical stimulation intensities affect the degree of striatal CBV decreases $\left(F_{(2,8)}=86.548, p<0.0001\right)$ despite the absence of differences between the two hemispheres (all $p>0.05)$. Fisher's post hoc tests revealed that the number of responsive pixels in the $\mathrm{CPu}$ for forepaw stimulation was larger at $60 \mathrm{~V}$ than at $5 \mathrm{~V}(p<0.0001)$ and $20 \mathrm{~V}(p<0.0001)$, and larger at $20 \mathrm{~V}$ than at $5 \mathrm{~V}(p<0.01)$. Figure $4 C$ shows that the number of responsive pixels in the S1FL was lower for $60 \mathrm{~V}$ stimulation than for $5 \mathrm{~V}$ stimulation $(p<0.05)$.
Neural activity of the CPu induced by nociceptive electrical stimulation

Fos immunohistochemistry and neural recording were used to identify whether the $\mathrm{CBV}$ decreases in the $\mathrm{CPu}$ was associated with changes in neuronal activity. Photomicrographs of Fos expression in the $\mathrm{CPu}$ for the control, formalin, and $60 \mathrm{~V}$ groups are shown in Figure $5 A$. A two-way ANOVA $(n=15)$ showed that the treatment exerted a main effect on the amount of Fos expression in the $\mathrm{CPu}\left(F_{(2,19)}=33.884, p<0.0001\right)$. Fisher's post hoc tests revealed that the level of Fos expression was higher in the 60 $\mathrm{V}$ group than in the control $(p<0.0001)$ and formalin $(p<$ $0.0001)$ groups, and higher in the formalin than in the control group $(p<0.05)$ (Fig. $5 B$ ). The Fos expression did not differ between the ipsilateral and contralateral $\mathrm{CPu}$ (all $p>0.05)$.

Neural electrophysiological signals were assessed as a second measure of the neural activity in the $\mathrm{CPu}$ induced by noxious electrical stimulation $(n=7)$. The recording sites are shown in Figure $5 C$. Traces recorded in the two hemispheres of the $\mathrm{CPu}$ are depicted in Figure 5D. Consistent with the Fos results, excitatory responses (corresponding to increased firing rates in spikes per second) were evident in the two hemispheres of the CPu during the stimulation. Two-tailed paired $t$ tests revealed that the averaged firing rate in the $\mathrm{CPu}$ was considerably higher than the baseline for $60 \mathrm{~V}$ electrical stimulation $(p<0.00001)$, and that the responses did not differ between the two hemispheres (Fig. $5 E)$. Both the Fos immunohistochemistry and electrophysiolog- 

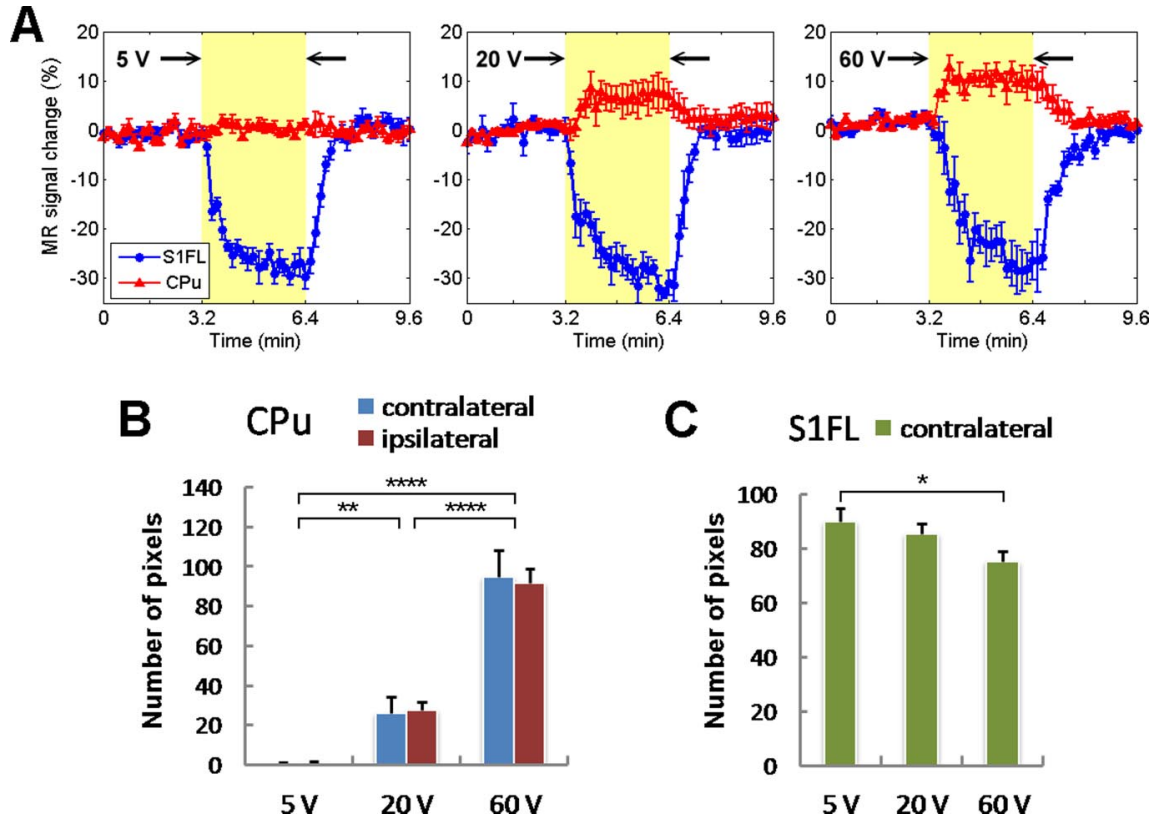

Figure 4. MR signal changes and the number of responsive pixels during graded electrical stimulation. $\boldsymbol{A}$, The signal-to-time curves were computed from the MR signals in the CPu and S1FL, averaged across five rats, and expressed as percentages relative to baseline values. The yellow-shaded regions indicate stimulus epochs. The $60 \mathrm{~V}$ stimulation induced the highest MR signal increase in the CPu, which can be correlated with the CBV decreases, whereas the contralateral S1FL showed CBV increases. $\boldsymbol{B}$, The number of pixels with significant MR signal increases $(p<0.05)$ was quantified in the $C P u$ of both hemispheres. No difference was found between the ipsilateral and contralateral CPu for all stimulation intensities. The $60 \mathrm{~V}$ stimulation induced the largest number of responsive pixels, followed by 20 and 5 V. C, The number of pixels with significant MR signal decreases was quantified in the contralateral S1FL. The activation area was significantly smaller for $60 \mathrm{~V}$ stimulation than for $5 \mathrm{~V}$ stimulation. ${ }^{*} p<0.05$ ${ }^{* *} p<0.01,{ }^{* * * *} p<0.0001$, significant. Error bars represent SEM values.

ical recordings indicated that the $60 \mathrm{~V}$ stimulation leading to CBV decreases in the $\mathrm{CPu}$ was associated with significantly higher neuronal activity in the region.

Effects of dopamine $\mathrm{D}_{2}$ antagonist on striatal $\mathrm{CBV}$ decreases D2DRs crucially affect CBV decreases in the CPu (Choi et al., 2006). Consequently, a D2DR antagonist, eticlopride, was used to investigate the possible role of D2DRs in modulating striatal vascular responses to noxious electrical stimulation. Identical imaging procedures were applied to five rats, with $60 \mathrm{~V}$ forepaw stimulation applied before and after the intravenous administration of $1 \mathrm{mg} / \mathrm{kg}$ eticlopride (Fig. $6 A$ ). Figure $6 B$ clearly illustrates the blocking effects of eticlopride. Two-tailed paired $t$ tests indicated that the administration of eticlopride significantly decreased the number of responsive pixels showing CBV decreases in the bilateral $\mathrm{CPu}(p<0.05)$, but had no significant effect in the contralateral S1FL. In addition, the use of saline as a vehicle control induced no observable changes (data not presented, $n=5$ ). These results suggest that D2DRs are a major mediator of CBV decreases in the $\mathrm{CPu}$ induced by noxious electrical stimulation.

\section{Discussion}

The present study demonstrates the novel finding that noxious electrical stimulation induces bilateral CBV decreases in the $\mathrm{CPu}$, which is associated with increased neural activity in the region. This finding is intriguing because it reveals at least three novel aspects: (1) that endogenous neurotransmission-related CBV decreases can lead to reduced CBV and hemodynamic responses in a region, (2) that CBV decreases can be associated with increased neuronal activity, despite some previous studies suggesting that decreased cerebral blood flow $(\mathrm{CBF})$ is associated with the inhi- bition of brain activity (Shmuel et al., 2002; Shmuel et al., 2006), and (3) that striatal CBV decreases modulated by D2DRs is an important mechanism that occurs in neurovascular processing of nociception.

CBV decreases can be intuitively ascribed to two major effects: (1) a reduction in CBF due to the suppression of neuronal activity (Shmuel et al., 2002, 2006), and (2) a "vascular-steal" effect, whereby a large amount of the blood is diverted to activated areas, leaving adjacent regions with a lower blood supply (Harel et al., 2002; Shmuel et al., 2002). The first effect cannot account for the observed CBV decreases in the present study since both our results (Fig. 5) and previous data indicate the occurrence of neuronal activation in the $\mathrm{CPu}$ during noxious stimulation (Chudler and Dong, 1995; Chudler, 1998). Nevertheless, the vascular-steal effect is also unlikely to explain our findings for three reasons: (1) no obvious CBV decreases were observed in regions neighboring the S1FL, (2) only a small proportion of the arterial blood supply to the sensory cortices and $\mathrm{CPu}$ is shared (Scremin, 1995), and (3) in the graded stimulation study, the level of CBV increases in the S1FL did not match that of CBV decreases in the $\mathrm{CPu}$ (Fig. 4C), indicating that the reduction in $\mathrm{CBV}$ in the $\mathrm{CPu}$ does not simply result from the reallocation of blood resources. In addition, inflow blood probably had minimal effects on the present results for the following two reasons: First, all the vessels, including arteries, veins, and capillaries, showed extremely low MR signals following injection of iron oxide contrast agent, which was due to the SPIOs inducing strong dephasing of MR signals (Wu et al., 2004). Second, the CBV-weighted MR images were acquired using a $\mathrm{T}_{2}{ }^{\star}$-weighted FLASH sequence, which would minimize the flow-related enhancement at a small flip angle (Keller et al., 1989).

The bilateral CBV decreases in the $\mathrm{CPu}$ are intriguing and warrant further investigation. This response might be mediated by a nociceptive pathway that bifurcates as it ascends from the periphery to the brain. In the present study, the electrical stimulation was applied to the right side, which activated the ipsilateral spinal sensory neurons in the dorsal root ganglia, as shown in Figure 1 . The projections of the spinal neurons directly or indirectly target central regions such as the thalamus (Willis and Westlund, 1997; Willis, 2007) and the substantia nigra (SN) (Chudler and Dong, 1995). The projections to the thalamus cross the midline whereas those to many other regions such as the SN bifurcate as they ascend. The $\mathrm{CPu}$ is a major target of $\mathrm{SN}$ projections, and hence bilateral responses were evident in the CBVweighted images, whereas S1FL responses were only evident on the contralateral side due to the S1FL receiving inputs from the thalamus.

The positive CBV response in the S1FL is mainly attributed to increases in neuronal activity and regional CBF. This mechanism is central to BOLD fMRI techniques and has been used to interpret most fMRI data; however, the negative CBV response in the $\mathrm{CPu}$ cannot be ascribed to this phenomenon. The signal sources 

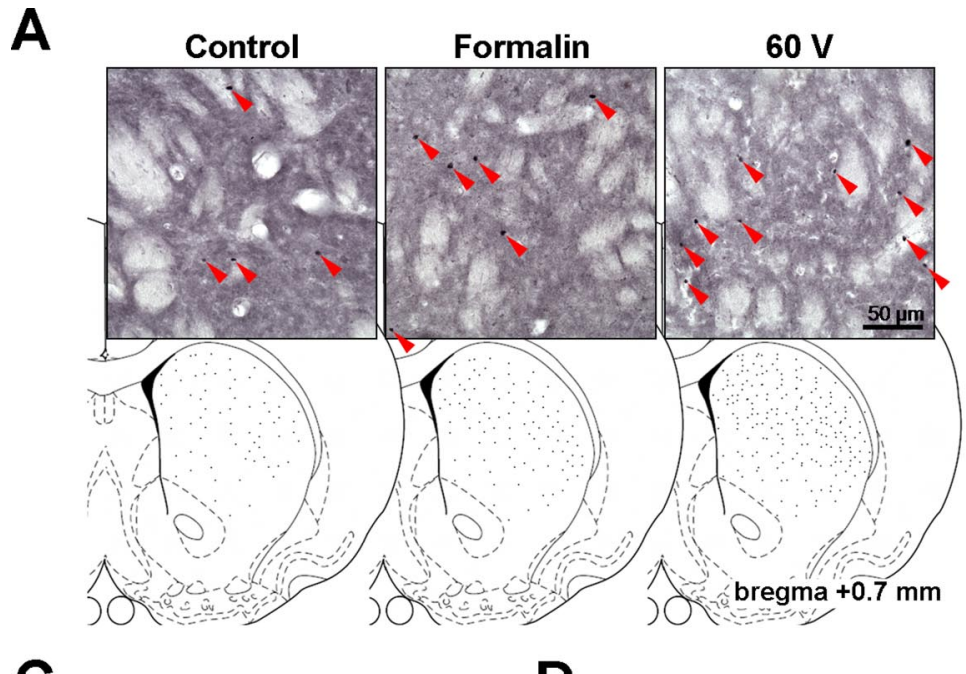

C

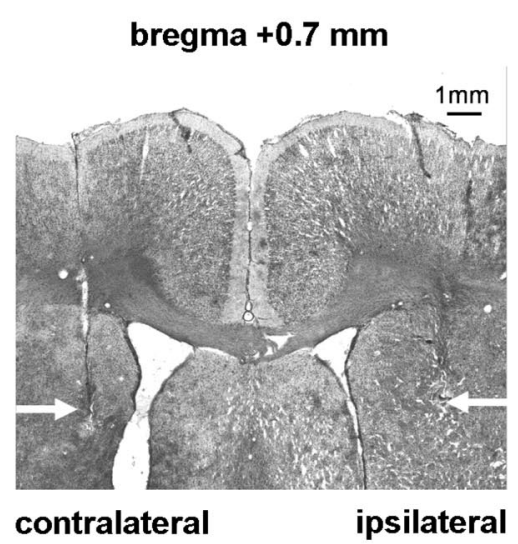

D
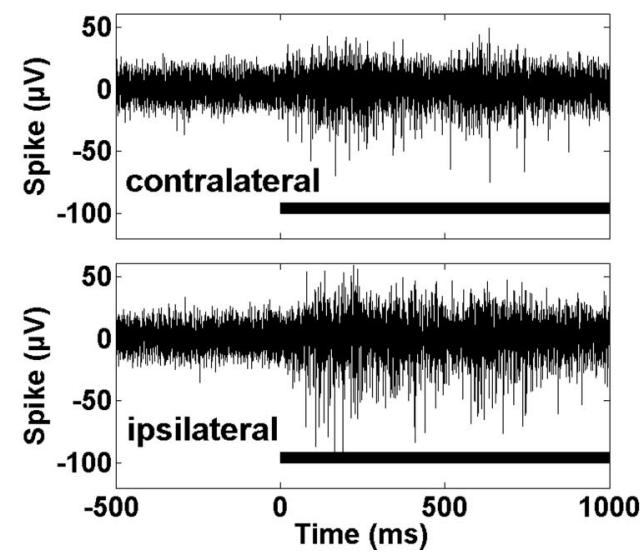

B

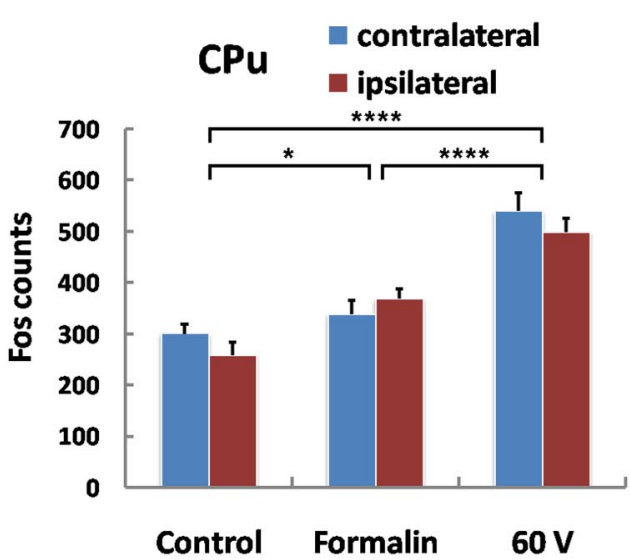

E
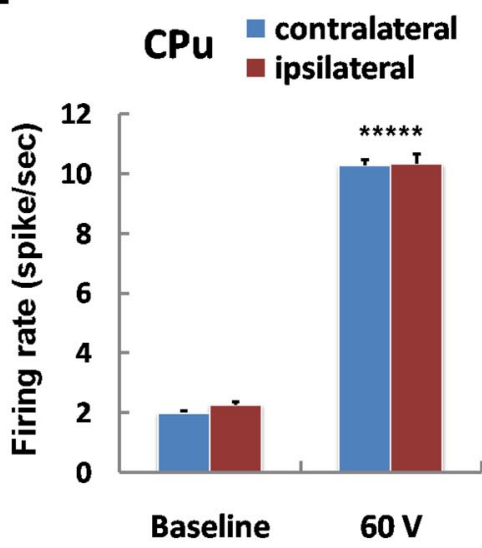

Figure 5. Striatal neuronal activity was investigated by Fos immunohistochemistry and electrophysiological recording in response to noxious electrical stimulation that produced CBV decreases in the region. $\boldsymbol{A}$, Photomicrographs (top) of Fos-ir cells and macro schematic drawings (bottom) of Fos-ir cell distribution from each treatment group. Arrowheads indicate Fos-ir cells. $\boldsymbol{B}$, Stimulation at $60 \mathrm{~V}$ induced the highest level of Fos expression, followed by formalin and control. C, Recording electrodes implanted into the ipsilateral and contralateral CPu shown in a Nissl-stained brain slice. The tips of the electrodes are indicated by white arrows. $D$, Traces of neural activity in the ipsilateral and contralateral CPu before and during electrical stimulation at $60 \mathrm{~V}$. The duration of the stimulus is indicated by the black bar. $\boldsymbol{E}$, The firing rate was significantly higher during stimulation than at the baseline. Both Fos immunohistochemistry and electrophysiological recording indicate that noxious electrical stimulation enhanced the neuronal activity in the CPu. ${ }^{*} p<0.05,{ }^{* * * *} p<0.0001,{ }^{* * * * *} p<0.00001$, significant. Error bars represent SEM values.
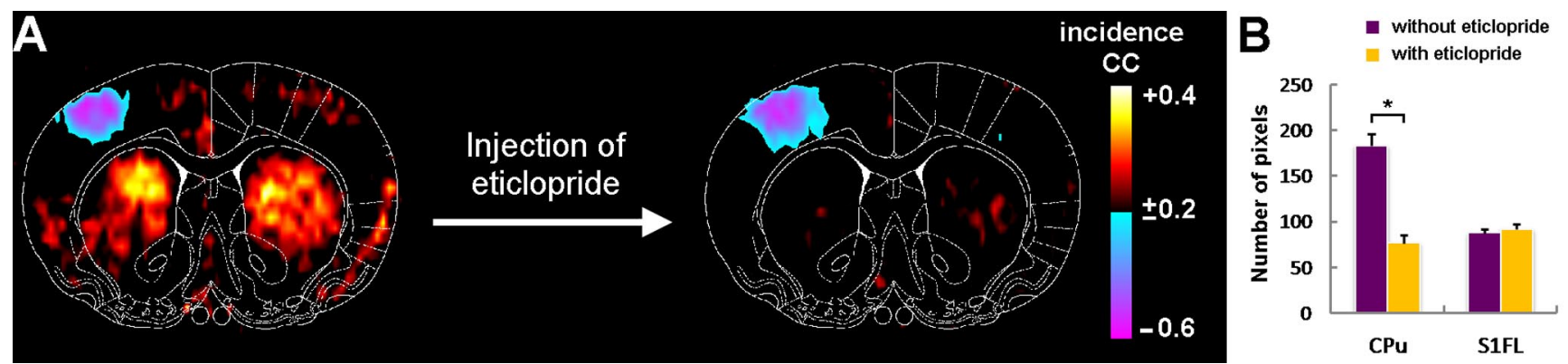

Figure 6. Effects of eticlopride, a D2DR antagonist, on striatal CBV decreases. $A$, Five rats received $60 \mathrm{~V}$ forepaw electrical stimulation followed by CBV-weighted fMRI at bregma $+0.7 \mathrm{~mm}$. CC data were used to generate the incidence maps, which show that CBV decreases in the bilateral CPu could be blocked by the intravenous injection of $1 \mathrm{mg} / \mathrm{kg}$ eticlopride. $\boldsymbol{B}$, Two-tailed paired $t$ tests indicate that eticlopride significantly reduced the number of responsive pixels in the CPu but had no effect in the S1FL. ${ }^{*}<0.05$, significant. Error bars represent SEM values.

have different origins and the level of neurotransmissioninduced CBV changes may be lower, which could result in a large difference in the signal amplitude between the positive CBV response in the S1FL and the negative CBV response in the $\mathrm{CPu}$. In another aspect, the effects of D2DRs in the cortex cannot be ignored since there is still a small amount of D2DRs in the cortical region (Chen et al., 2005; Choi et al., 2006). When dopaminergic neurotransmission occurs in the cortex, a negative fMRI response might also be detected depending on the amount of D2DRs or the strength of D2DR-induced CBV decreases. Note that Figure $4 C$ shows that the number of responsive pixels in S1FL images decreased with increasing stimulation intensity, which is probably due to the activation of cortical D2DRs counteracting the CBV increases. 
Endogenous neurotransmission-induced CBV decreases might counteract the increase in regional CBF due to enhanced neuronal activity, thus resulting in negative or reduced BOLD responses. This hypothesis could explain the difficulty of finding nociception-induced hemodynamic responses in the $\mathrm{CPu}$ in both humans and rodents (Casey, 1999; Tuor et al., 2000; Chang and Shyu, 2001; Farrell et al., 2005; Lowe et al., 2007), and a recent report of negative $\mathrm{BOLD}$ responses in the $\mathrm{CPu}$ induced by intense electrical stimulation to the rat forepaw (Zhao et al., 2008a). Moreover, consistent with our findings, Morrow et al. reported that $\mathrm{CBF}$ was reduced during the early phase of formalin-induced pain (Morrow et al., 1998). The reduced hemodynamic responses, which were not explained by those authors, might be due to the presence of D2DR-mediated CBV decreases in the CPu. Therefore, the involvement of vasoactive neurotransmitters should be considered an important determinant of CBV, CBF, or BOLD responses.

The role of the D2DR and dopaminergic system has long been investigated in nociceptive processing. There is evidence that striatal dopaminergic neurotransmission is specifically involved in antagonizing nociceptive responses (Hagelberg et al., 2004; Mansikka et al., 2005; Martikainen et al., 2005; Pelissier et al., 2006), since lesioning striatal dopaminergic neurons increases pain sensitivity, whereas activating these neurons inhibits pain responses (Lin et al., 1984). Furthermore, the infusion of D2DR agonists and antagonists reduced and augmented nociception, respectively. In contrast, no changes in pain-related behavior were observed following up-regulation or down-regulation of dopamine D1 receptor (D1DR) activity (Magnusson and Fisher, 2000). These findings suggest that striatal neurons expressing D2DRs are major players in antinociceptive effects on pain modulation. Nevertheless, this perspective is probably oversimplified since neuronal cells are not the sole participants in brain functioning (Park et al., 2003; Iadecola, 2004; Lok et al., 2007).

The activity of neuronal cells is highly dependent on the energy and metabolite supply in the microvasculature and neuronal activation produces changes in the local CBF. These reciprocal actions of neurons and microvasculature on each other indicate the importance of considering the two components as a functional unit in the execution of brain functions. It is paradoxical that neuronal activation is associated with CBV decreases. An emerging concept holds that neural cells and the respective microvasculature cooperate in concert as an integrated unit to perform brain functions. The two components might be influenced mutually or together by factors present in the unit, such as neurotransmitters. Neuronal activation increases the local blood flow under many conditions, which underlies the central principle of hemodynamics-based neuroimaging techniques. However, as shown by the present study, the presence of vasoactive neurotransmitters such as dopamine in the neurovascular unit can significantly alter neurovascular responses (Choi et al., 2006).

It is likely that the CBV decreases associated with neuronal activation is due to the modulation of dopamine release induced by nociception occurring in the neurovascular units of the $\mathrm{CPu}$. Within these units, neurons with dopaminergic receptors are activated in response to dopamine release, whereas the microvessels, which are closely apposed to dopaminergic fibers (Krimer et al., 1998), are constricted by the actions of dopamine. It cannot be ruled out that CBV increases induced by local striatal neuronal activation or D1DR is also activated by the released dopamine. However, these effects appeared to be insignificant compared with the $\mathrm{D}_{2}$-related responses.

The role of dopamine-mediated CBV decreases in the CPu in the modulation of nociception remains obscure. The effects of blood perfusion act over millimeter distances (Park et al., 2003), and thus can serve as a general mechanism for efficiently adapting the microenvironment for neuronal functioning. However, it is intriguing that increased neuronal activity is matched to a functional context, whereby vascular metabolism is down-regulated by CBV decreases. One possible explanation is that the activated neurons respond directly to pain modulation, while striatal CBV decreases has a low energy tone to maintain the quiescent neural activity. Such paradoxical neurovascular coordination should be considered as a critical characteristic of brain regions that receive modulation of vasoactive neurotransmission.

In conclusion, the present study demonstrates the existence of a nociception-induced bilateral CBV decreases in the CPu associated with increased neuronal activity. Although the actual role of striatal CBV decreases in pain modulation warrants further investigations, the discovery of a combined response involving CBV decreases and increased neural activity to nociception leads to several interesting questions related to both the underlying neurovascular mechanism and pain research. Our findings suggest that the endogenous release of vasoactive neurotransmitters, depending on the complex interplay among the neuronal circuitry, markedly affects CBV-weighted fMRI signals. Attention should be paid to the influence of endogenous neurotransmission when interpreting negative BOLD responses.

\section{References}

Casey KL (1999) Forebrain mechanisms of nociception and pain: analysis through imaging. Proc Natl Acad Sci U S A 96:7668-7674.

Chang C, Shyu BC (2001) A fMRI study of brain activations during nonnoxious and noxious electrical stimulation of the sciatic nerve of rats. Brain Res 897:71-81.

Chen YC, Choi JK, Andersen SL, Rosen BR, Jenkins BG (2005) Mapping dopamine D2/D3 receptor function using pharmacological magnetic resonance imaging. Psychopharmacology 180:705-715.

Choi JK, Chen YI, Hamel E, Jenkins BG (2006) Brain hemodynamic changes mediated by dopamine receptors: role of the cerebral microvasculature in dopamine-mediated neurovascular coupling. Neuroimage 30:700-712.

Chudler EH (1998) Response properties of neurons in the caudateputamen and globus pallidus to noxious and non-noxious thermal stimulation in anesthetized rats. Brain Res 812:283-288.

Chudler EH, Dong WK (1995) The role of the basal ganglia in nociception and pain. Pain 60:3-38.

Devor A, Tian P, Nishimura N, Teng IC, Hillman EM, Narayanan SN, Ulbert I, Boas DA, Kleinfeld D, Dale AM (2007) Suppressed neuronal activity and concurrent arteriolar vasoconstriction may explain negative blood oxygenation level-dependent signal. J Neurosci 27:4452-4459.

Donoho DL, Johnstone IM (1994) Ideal spatial adaptation by wavelet shrinkage. Biometrika 81:425-455.

Farrell MJ, Laird AR, Egan GF (2005) Brain activity associated with painfully hot stimuli applied to the upper limb: a meta-analysis. Hum Brain Mapp 25:129-139.

Hagelberg N, Jääskeläinen SK, Martikainen IK, Mansikka H, Forssell H, Scheinin H, Hietala J, Pertovaara A (2004) Striatal dopamine D2 receptors in modulation of pain in humans: a review. Eur J Pharmacol 500:187-192.

Harel N, Lee SP, Nagaoka T, Kim DS, Kim SG (2002) Origin of negative blood oxygenation level-dependent fMRI signals. J Cereb Blood Flow Metab 22:908-917.

Iadecola C (2004) Neurovascular regulation in the normal brain and in Alzheimer's disease. Nat Rev 5:347-360.

Keilholz SD, Silva AC, Raman M, Merkle H, Koretsky AP (2006) BOLD and $\mathrm{CBV}$-weighted functional magnetic resonance imaging of the rat somatosensory system. Magn Reson Med 55:316-324.

Keller PJ, Drayer BP, Fram EK, Williams KD, Dumoulin CL, Souza SP (1989) MR angiography with two-dimensional acquisition and threedimensional display. Work in progress. Radiology 173:527-532.

Krimer LS, Muly EC 3rd, Williams GV, Goldman-Rakic PS (1998) Dopami- 
nergic regulation of cerebral cortical microcirculation. Nat Neurosci $1: 286-289$.

Lin MT, Wu JJ, Tsay BL (1984) Effects of kainic acid injections in the striatum on physiologic and behavioral functions in conscious rats. Exp Neurol 83:71-83.

Liu ZM, Schmidt KF, Sicard KM, Duong TQ (2004) Imaging oxygen consumption in forepaw somatosensory stimulation in rats under isoflurane anesthesia. Magn Reson Med 52:277-285.

Lok J, Gupta P, Guo S, Kim WJ, Whalen MJ, van Leyen K, Lo EH (2007) Cell-cell signaling in the neurovascular unit. Neurochem Res 32:2032-2045.

Lowe AS, Beech JS, Williams SC (2007) Small animal, whole brain fMRI: innocuous and nociceptive forepaw stimulation. Neuroimage $35: 719-728$.

Magnusson JE, Fisher K (2000) The involvement of dopamine in nociception: the role of $\mathrm{D}(1)$ and $\mathrm{D}(2)$ receptors in the dorsolateral striatum. Brain Res 855:260-266.

Mandeville JB, Marota JJ, Kosofsky BE, Keltner JR, Weissleder R, Rosen BR, Weisskoff RM (1998) Dynamic functional imaging of relative cerebral blood volume during rat forepaw stimulation. Magn Reson Med 39:615-624.

Mansikka H, Erbs E, Borrelli E, Pertovaara A (2005) Influence of the dopamine $\mathrm{D} 2$ receptor knockout on pain-related behavior in the mouse. Brain Res 1052:82-87.

Martikainen IK, Hagelberg N, Mansikka H, Hietala J, Någren K, Scheinin H, Pertovaara A (2005) Association of striatal dopamine D2/D3 receptor binding potential with pain but not tactile sensitivity or placebo analgesia. Neurosci Lett 376:149-153.

Morrow TJ, Paulson PE, Danneman PJ, Casey KL (1998) Regional changes in forebrain activation during the early and late phase of formalin nociception: analysis using cerebral blood flow in the rat. Pain 75:355-365.

Ogawa S, Lee TM, Kay AR, Tank DW (1990) Brain magnetic resonance imaging with contrast dependent on blood oxygenation. Proc Natl Acad Sci U S A 87:9868-9872.

Park JA, Choi KS, Kim SY, Kim KW (2003) Coordinated interaction of the vascular and nervous systems: from molecule- to cell-based approaches. Biochem Biophys Res Commun 311:247-253.

Paxinos G, Watson C (1998) The rat brain in stereotaxic coordinates: San Diego: Academic.

Pelissier T, Laurido C, Hernandez A, Constandil L, Eschalier A (2006) Biphasic effect of apomorphine on rat nociception and effect of dopamine D2 receptor antagonists. Eur J Pharmacol 546:40-47.

Pertovaara A, Martikainen IK, Hagelberg N, Mansikka H, Någren K, Hietala J, Scheinin H (2004) Striatal dopamine D2/D3 receptor availability correlates with individual response characteristics to pain. Eur J Neurosci 20:1587-1592.

Schridde U, Khubchandani M, Motelow JE, Sanganahalli BG, Hyder F, Blumenfeld H (2008) Negative BOLD with large increases in neuronal activity. Cereb Cortex 18:1814-1827.

Scott DJ, Heitzeg MM, Koeppe RA, Stohler CS, Zubieta JK (2006) Varia- tions in the human pain stress experience mediated by ventral and dorsal basal ganglia dopamine activity. J Neurosci 26:10789-10795.

Scremin OU (1995) Cerebral vascular system. In: The rat nervous system (Paxions G, ed), pp 3-35. San Diego: Academic.

Shih YY, Chen YY, Chen JC, Chang C, Jaw FS (2007) ISPMER: integrated system for combined PET, MRI, and electrophysiological recording in somatosensory studies in rats. Nucl Instrum Methods Phys Res A 580:938-943.

Shih YY, Chang C, Chen JC, Jaw FS (2008a) BOLD fMRI mapping of brain responses to nociceptive stimuli in rats under ketamine anesthesia. Med Eng Phys 30:953-958.

Shih YY, Chen YY, Chen CC, Chen JC, Chang C, Jaw FS (2008b) Wholebrain functional magnetic resonance imaging mapping of acute nociceptive responses induced by formalin in rats using atlas registration-based event-related analysis. J Neurosci Res 86:1801-1811.

Shih YY, Chiang YC, Chen JC, Huang CH, Chen YY, Liu RS, Chang C, Jaw FS $(2008 \mathrm{c})$ Brain nociceptive imaging in rats using (18)ffluorodeoxyglucose small-animal positron emission tomography. Neuroscience 155:1221-1226.

Shmuel A, Yacoub E, Pfeuffer J, Van de Moortele PF, Adriany G, Hu X, Ugurbil K (2002) Sustained negative BOLD, blood flow and oxygen consumption response and its coupling to the positive response in the human brain. Neuron 36:1195-1210.

Shmuel A, Augath M, Oeltermann A, Logothetis NK (2006) Negative functional MRI response correlates with decreases in neuronal activity in monkey visual area V1. Nat Neurosci 9:569-577.

Tuor UI, Malisza K, Foniok T, Papadimitropoulos R, Jarmasz M, Somorjai R, Kozlowski P (2000) Functional magnetic resonance imaging in rats subjected to intense electrical and noxious chemical stimulation of the forepaw. Pain 87:315-324.

Tuor UI, McKenzie E, Tomanek B (2002) Functional magnetic resonance imaging of tonic pain and vasopressor effects in rats. Magn Reson Imaging 20:707-712.

Van Camp N, Verhoye M, Van der Linden A (2006) Stimulation of the rat somatosensory cortex at different frequencies and pulse widths. NMR Biomed 19:10-17.

Willis WD, Westlund KN (1997) Neuroanatomy of the pain system and of the pathways that modulate pain. J Clin Neurophysiol 14:2-31.

Willis WD Jr (2007) The somatosensory system, with emphasis on structures important for pain. Brain Res Rev 55:297-313.

Wu EX, Tang H, Jensen JH (2004) Applications of ultrasmall superparamagnetic iron oxide contrast agents in the MR study of animal models. NMR Biomed 17:478-483.

Zhao F, Zhao T, Zhou L, Wu Q, Hu X (2008a) BOLD study of stimulationinduced neural activity and resting-state connectivity in medetomidinesedated rat. Neuroimage 39:248-260.

Zhao F, Williams M, Meng X, Welsh DC, Coimbra A, Crown ED, Cook JJ, Urban MO, Hargreaves R, Williams DS (2008b) BOLD and blood volume-weighted fMRI of rat lumbar spinal cord during non-noxious and noxious electrical hindpaw stimulation. Neuroimage 40:133-147. 